\title{
Eliciting steady-state visual evoked potentials by means of stereoscopic displays
}

\author{
Enrico Calore, Davide Gadia, Daniele Marini, \\ Dipartimento di Informatica, Università degli Studi di Milano, Via Comelico 39, 20135 Milano, \\ Italy
}

\begin{abstract}
Brain-Computer Interfaces (BCIs) provide users communication and control capabilities by analyzing their brain activity. A technique to implement BCIs, used recently also in Virtual Reality (VR) environments, is based on the Steady State Visual Evoked Potentials (SSVEPs) detection. Exploiting the SSVEP response, BCIs could be implemented showing targets flickering at different frequencies and detecting which is gazed by the observer analyzing her/his electroencephalographic (EEG) signals. In this work, we evaluate the use of stereoscopic displays for the presentation of SSVEP eliciting stimuli, comparing their effectiveness between monoscopic and stereoscopic stimuli. Moreover we propose a novel method to elicit SSVEP responses exploiting the stereoscopic displays capability of presenting dichoptic stimuli. We have created an experimental scene to present flickering stimuli on an active stereoscopic display, obtaining reliable control of the targets' frequency independently for the two stereo views. Using an EEG acquisition device, we analyzed the SSVEP responses from a group of subjects. From the preliminary results, we got evidence that stereoscopic displays represent valid devices for the presentation of SSVEP stimuli. Moreover, the use of different flickering frequencies for the two views of a single stimulus proved to elicit non-linear interactions between the stimulation frequencies, clearly visible in the EEG signal. This suggests interesting applications for SSVEP-based BCIs in VR environments able to overcome some limitations imposed by the refresh frequency of standard displays, but also the use of commodity stereoscopic displays to implement binocular rivalry experiments.
\end{abstract}

Keywords: Stereoscopy, Virtual Reality, Brain-Computer Interface, EEG, Steady-state visual evoked potential, binocular rivalry

\section{INTRODUCTION}

A Brain-Computer Interface (BCI) is defined as a direct communication pathway between the brain and an external device as a computer or a machine in general ${ }^{1}$. It can be implemented using different kind of devices capable of reading the brain activity of an user and signal processing algorithms capable of extrapolate from the read activity a particular state of the user's brain. To each detectable state could be associated a command for an actuator.

Most of the research regarding BCIs aimed to provide mobility-impaired users with a tool capable of translating a thought or a will to a command for an external device or a prosthetic limb. More recently, thanks to the advancement of the research in the field both from the software and from the hardware point of view, BCIs use is being investigated also in the field of human-computer interaction to provide new communication channels that do not relay on the user's limb movements.

BCIs could be implemented using various modalities. One of the most used for its robustness is based on the Steady-State Visual Evoked Potential (SSVEP) detection: when a visual stimulus flickering at a constant frequency is gazed by an user, it is possible to detect in her/his Electroencephalogram (EEG) a continuous brain response at the corresponding frequency.

Exploiting the SSVEP response, SSVEP-based BCIs could be implemented showing to the user targets flickering at different frequencies and detecting which is gazed by the observer, associating to each of them a command.

Authors E-mail: \{enrico.calore, davide.gadia, daniele.marini\}@unimi.it 
BCIs are recently gaining attention in fields like computer gaming and VR environments interaction. In particular, SSVEP-based BCIs have already been successfully used, integrating flickering targets, in VR environments $^{2,3}$. Consequently, as stereoscopic displays are becoming widely available and often adopted for 3D VR applications, it is interesting to evaluate their use to present SSVEP eliciting stimuli and the possibilities they may offer to provide better SSVEP-based BCIs.

\section{STEADY-STATE VISUAL EVOKED POTENTIALS BASED BCI}

An Evoked Potential (EP), in the context of EEG signals, is an electrical potential elicited by the presentation of a stimulus that can be recorded from the nervous system. In particular, in the case of non-invasive EEG recordings, it can be acquired from an electrode positioned on the surface of the scalp. Visual Evoked Potentials (VEP) are EP elicited by a visual stimulation.

A steady-state VEP (SSVEP) is a particular VEP, where the stimulus is presented several times at a frequency at least higher than $3.5 \mathrm{~Hz}$, but more commonly higher than $6 \mathrm{~Hz}$. In this case, a periodic response called SSVEP can be observed in the recorded scalp EEG signal, in particular in the occipital brain region, where the visual cortex resides.

The SSVEP response has been studied deeply in the field of vision research starting from the seventies ${ }^{4}$, but it has been often investigated and exploited in the fields of cognitive neuroscience and clinical neuroscience until today $^{5}$. Moreover, in the last years, it has been adopted widely for the implementation of BCIs ${ }^{5,6}$.

The SSVEP modality has been widely adopted because of its high level of detection accuracy, the short calibration time needed, the low number of EEG electrodes required and also for the low BCI illiteracy showed ${ }^{7}$, granting high usability for most of the users, also in out-of-laboratory environments.

The stimulus presented to elicit a SSVEP response is commonly a flickering light and accordingly to the flickering frequency, an increase in the power of the signal, at the corresponding frequency, can be detected in the EEG signal acquired from the scalp of an user gazing at the stimulus. Consequently, in the case of SSVEP, the presence of the response can be detected analyzing the frequency spectrum of the recorded signal.

In presence of multiple stimuli flickering at different frequencies in the visual field of the user (often referred as frequency tagged SSVEP-based BCI), the increase of the signal power in the frequencies of the stimuli is more pronounced in the single frequency corresponding to the stimulus the user is attending to. Therefore, BCIs can be implemented using this modality, since the user, attending to a particular stimulus chosen from the presented ones, can communicate to the system to execute a particular operation previously associated to the stimulus.

Stimuli are commonly presented by LED lights, or by shapes on a regular computer monitor, flickering at frequencies ranging between $6 \mathrm{~Hz}$ and $40 \mathrm{~Hz}$ (although it has been proven that SSVEP can be detected also for higher frequencies ${ }^{8}$ ).

The integration of the SSVEP stimuli presentation in Virtual Environments (VE), in contrast to the use of external stimuli generators as LED lights, is known to enrich the user presence and immersivity ${ }^{2,3}$, but it also introduces various technical limitations. First of all, the stimuli frequencies have to be divisors of the screen refresh frequency (commonly set to $60 \mathrm{~Hz}$ ) in order to provide a frequency-stable flickering stimulus. ${ }^{9}$ Moreover, software tools are needed to provide an accurate stimuli synchronization with the screen refresh ${ }^{5}$.

\section{SSVEP-BASED BCI AND STEREOSCOPIC VR}

Stereoscopic visualization in cinematography and VR creates an illusion of depth by means of two bidimensional images corresponding to different views of a scene.

This illusion is based on making believe to the Human Visual System (HVS) that the two different images correspond to the two different perspective views captured by the eyes. The two images commonly contains objects which, according to their depth, present different horizontal disparities between the two views.

The introduction of stereoscopy in the production of movies or of VR environments allows an observer to enhance the sense of immersivity and presence. In the context of VR installations, stereoscopic devices are 
indeed widely used and recently they were introduced also in the end-user market as stereoscopic televisions and computer monitors.

The use of BCI systems in conjunction with VR environments demonstrated to produce various benefits ${ }^{2}$. VR environments are often presented to the users by means of sterescopic displays; nevertheless, very few studies are available about the influence that stereoscopic displays may have on the SSVEP stimuli presentation and consequently on the SSVEP elicitation and detection.

An interesting question regarding these display devices, is whether or not they could be used to integrate SSVEP stimuli inside the presented stereoscopic VE, and, more interestingly, if their capability of showing two independent images to the user's eyes could be exploited to enhance SSVEP-based BCIs performances.

SSVEP-based BCIs were implemented in the past also using stereoscopic displays, thus it is known to be possible to use them to this aim, but few studies compared the same BCI both for monoscopic and stereoscopic stimuli presentations concerning the effectiveness of the elicited SSVEP response. Moreover, as far as we know, there are no research works trying to exploit them to provide dichoptic stimuli* aimed to implement better SSVEP-based BCIs. Dichoptic stimulation, both for VEP and SSVEP elicitation, was used mainly in the field of vision research, with the aim of studying how the depth perception given by binocular disparity is handled by the $\mathrm{HVS}^{10}$.

In a very recent work $^{11}$ a SSVEP-based BCI, implementing a navigation task, has been tested, using a monoscopic and a stereoscopic VR environment. Some of the users performed better in the former condition, while others in the latter. Anyhow, interestingly the authors found a correlation between the two groups and their reported visual fatigue, highlighting the fact that the benefit of using a more immersive environment is counterbalanced by an increased visual fatigue for the group of most sensitive users.

An increased fatigue for the user can indeed have, as a consequence, a decrease in the attention towards the stimuli, which is known to be essential for a strong SSVEP elicitation ${ }^{5}$, thus provoking a decrease in the overall BCI performance.

In the first experiment we present in Section 5, our aim is indeed to study if a flickering stimulus presented with a non-zero sterescopic disparity, independently from any other depth cue, elicits a stronger or a weaker SSVEP response in the subjects. This would be an interesting information to take into account when planning SSVEP-based BCIs in the context of stereoscopic VR environments with integrated visual stimuli.

On the other side, in the second experiment presented in Section 6, our aim is to identify a possibility to exploit the capability of showing dichoptic stimuli, offered by stereoscopic displays, not only to generate the illusion of depth in the user, but also to overcome the limitation to the usable number of targets in a frequency tagged SSVEP-based BCI.

From previous experiments in the field of vision research, it is known that showing to a subject a stereoscopic target which changes repetitively its depth (i.e. its stereoscopic disparity) at a constant frequency, instead of its color or pattern, is possible to elicit a $\mathrm{VEP}^{10}$, and consequently probably a SSVEP as well. Despite of this, the amplitude of the evoked VEP was reported as being much smaller than using a color or pattern change, thus this seems not an efficient way to exploit sterescopic displays for better SSVE-based BCIs. Moreover, a change in stereoscopic disparity is commonly associated to muscular eye movements due to eyes vergence ${ }^{\dagger}$ which are known to produce EEG artifacts ${ }^{10}$, but also to easily elicit fatigue in the users.

A more interesting approach would be to use different stimulation frequencies for the two different stereoscopic views of the same target. If such frequencies combinations could be detected in the EEG signals, in principle, three different targets could be presented using only two frequencies $f_{1}$ and $f_{2}$, where target 1 flickers in both views at $f_{1}$, target 2 flickers in both views at $f_{2}$ while target 3 flickers at $f_{1}$ in one view and at $f_{2}$ in the other.

The use of a dichoptic stimulation using two different flickering frequencies for the same target should present in the EEG non-linear combinations of the used frequencies ${ }^{10,12}$. The use of this kind of stimulation was

${ }^{*}$ Dichoptic refers to viewing a separate and independent field by each eye. In dichoptic presentation, a stimulus A is presented to the left eye while a stimulus B is presented to the right eye.

${ }^{\dagger}$ Vergence is the simultaneous movement of both eyes to obtain or maintain single binocular vision. The eyes must rotate around a vertical axis so that the projection of the image is in the center of the retina in both eyes. 
indeed discussed in a vision research work investigating the detection of VEP non-linear components elicited by a dichoptic two frequency stimulation ${ }^{13}$. These kind of non-linear interactions were later studied using the bispectrum higher order spectral analysis ${ }^{14,15}$.

Thanks to these studies, as recalled also in ${ }^{10 \ddagger}$, it is known that showing to the left eye of a subject a flicker of frequency $f_{1}$ and to the right eye a flicker of frequency $f_{2}$, nonlinear processes produce harmonics of $f_{1}$ in left eye pathway and harmonics of $f_{2}$ in the right eye pathway. Moreover, nonlinear processes, occurring after the monocular signals are combined, produce cross-modulation terms of the general form $n f_{1}+m f_{2}$ for integral values of $n$ and $m$.

In the second experiment presented in this paper we therefore propose a novel approach to exploit stereoscopic displays to present this kind of dichoptic stimulation in order to overcome the limited number of targets presentable in a frequency tagged SSVEP-based BCI. Such a paradigm could be helpful also to use a limited set of frequencies known to elicit a strong SSVEP response, in order to present a larger set of targets.

\section{MATERIAL AND METHODS}

We have initially set up a software framework capable of presenting to the users flickering stimuli as custom objects in a stereoscopic VE scene to elicit a SSVEP response and to contextually record the users' EEG $^{16}$.

The OpenVibe software ${ }^{17}$ was used for the data acquisition, since it supports different acquisition devices and allows for an easy integration of custom signal processing algorithms and communications with other external software.

The OpenGL library ${ }^{18}$ was used for the scene rendering and stimuli presentation, in order to have an accurate timing and to allow for a complete customization of the scene, being able to handle separately the two different views for the two eyes. A custom OpenGL program has been written to access the lowest level synchronization functions available, starting from the results presented in ${ }^{19,20}$, to allow for accurate and stable flickering stimuli frequencies ${ }^{16}$.

Moreover, thanks to the flexibility of OpenVibe and to the GLFW library timing functions ${ }^{20}$, timestamps related to the stimuli presentation could be merged with the acquired EEG, allowing for more complex analysis for the detection of the SSVEP responses than the commonly used frequency filtering and energy estimation.

In particular it has been foreseen the possibility to perform phase analysis on the SSVEP response signals, as proposed $\mathrm{in}^{21}$, without the need of an external photodiode sensors, but implementing all the needed synchronizations between stimuli presentation and EEG signal acquisition in the software framework.

\section{1 g.Tec g.MOBIlab+ multipurpose}

To perform EEG recordings, for all the experiments described in the next Sections, we have used the g.Tec g.MOBIlab+ multipurpose version.

This is a portable biosignal acquisition device utilizable to acquire EEG, electrocardiogram (ECG), electrooculogram (EOG) and electromyogram (EMG) bio-signals. It is equipped with low-noise biosignal amplifiers and a 16 bit analog to digital converter sampling at $256 \mathrm{~Hz}$. It can be connected to a regular computer thanks to a Bluetooth connection for data acquisition. It has 8 channels as the regular version, but only four of them can be used to acquire EEG signals, since EEG, ECG and EMG signals have different amplitudes and therefore amplifiers with different gains have to be used (e.g. EEG signals commonly have an amplitude of several microvolts while ECG signals have an amplitude of few millivolts).

\footnotetext{
${ }^{\ddagger}$ In particular, Sec. 11.7 and Sec. 13.1.8b
} 


\section{FIRST EXPERIMENTAL SETUP}

Data acquisition was performed with the g.Tec g.MOBIlab+ multipurpose version described in Sec. 4.1 and the OpenVibe framework was used to store EEG traces to file, within stimulus start/end triggers, for off-line analysis.

Electrodes was placed on 4 scalp locations over the visual cortex, POz, Oz, O1, O2, referenced at the left ear lobe and grounded at Fpz according to the extended 10-20 system.

All the subjects performing the experiment where males between 25 and 36 years old which where tested and resulted to have normal stereoscopic view.

Using the custom developed software and an ASUS VG278 monitor with NVIDIA 3D Vision technology, a single $15 \mathrm{~Hz}$ flickering square white patch over a black background was shown to each subject.

The stereoscopic stimulus was shown for $8 \mathrm{~s}$ with zero disparity between the two views and then followed by $5 \mathrm{~s}$ interval without stimulus (i.e. only the black background was shown), then the sequence was repeated with the same stimulus, but with a positive disparity between the two views and later again with a negative disparity. The whole trial was then repeated 4 times for each subject.

Stimulus size was kept in the order of $6^{\circ}$ of visual angle adjusting the users distance from the monitor, while both positive and negative disparity where chosen to not induce eye strain, but anyhow to be relatively strong and thus in the order of $1^{\circ}$ of visual angle.

The SSVEP response was later computed for all the EEG epochs where a stimulation was present using the Minimum Energy Combination method and the $T$ test statistic ${ }^{22}$, which in the following sections will be referred to as $T$ index, to avoid confusions with the statistical t-test.

\subsection{Results}

The results of this experiment highlighted that a significant correlation between the stereoscopic disparity of the stimulus and the SSVEP response strength is not present.

Mean and standard deviation of the computed $T$ index values are reported in Tab. 1 where an inter-subject variability can be appreciated, but where the disparity between the two views of the stimulus seems not to influence the strength of the elicited SSVEP response.

\begin{tabular}{lcccccc}
\hline & \multicolumn{2}{c}{$\begin{array}{l}\text { Negative Disparity } \\
\text { Mean }\end{array}$} & Std & \multicolumn{2}{c}{ Zero Disparity } & \multicolumn{2}{c}{ Positive Disparity } \\
& Mean & Std & Mean & Std \\
\hline Subject 1 & 7.85 & 0.49 & 8.47 & 1.78 & 9.05 & 0.21 \\
Subject 2 & 4.83 & 0.78 & 3.54 & 0.36 & 5.82 & 0.61 \\
Subject 3 & 4.50 & 2.20 & 6.56 & 3.12 & 3.75 & 2.10 \\
Subject 4 & 4.13 & 1.10 & 3.66 & 0.95 & 4.10 & 0.30 \\
\hline
\end{tabular}

Table 1. SSVEP $T$ index computed for 4 subjects attending the same flickering stimulus at $15 \mathrm{~Hz}$ presented on a stereoscopic display with different disparities.

It is worth to notice that the stimulus size was kept fixed and only the disparity between the two views was changed, thus most of the users did not perceived any difference between the different disparity stimuli, apart from the sensation of the eyes moving due to vergence. It would be interesting to investigate if using other depth cues as objects size changes and perspective views, a conscious depth perception may influence the user attention and consequently the SSVEP response significantly.

Anyhow, aim of this experiment was to assess only the possible influences given by the dichoptic stimulus disparity. More complex setups would pose higher challenges in order to separate the effects of the different phenomena involved, since a stimulus size change would for sure influence the SSVEP response, due to a known relation between the stimulus field size and the SSVEP response ${ }^{23}$. 


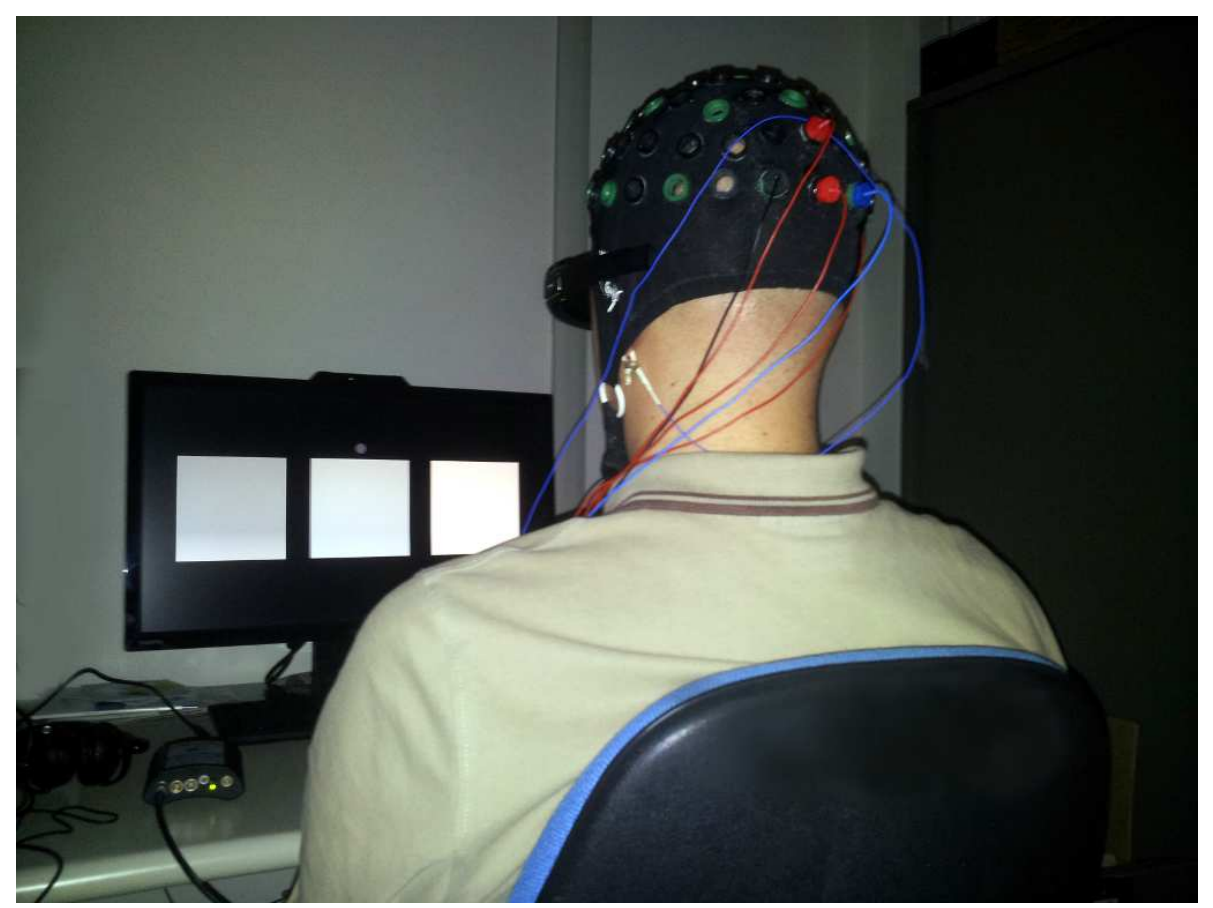

Figure 1. One subject performing the second experiment.

\section{SECOND EXPERIMENTAL SETUP}

In the second experiment the same hardware setup and electrode locations were adopted. The experimental scene presented three white patches placed side by side on a black background as shown in Fig. 1.

The left patch was flickering at $15 \mathrm{~Hz}$, while the right one at $20 \mathrm{~Hz}$ for both of the stereoscopic views. On the other hand, the patch in the center was presenting a dichoptic flicker: the left view of the patch was flickering at $15 \mathrm{~Hz}$, while the right view of the patch was flickering at $20 \mathrm{~Hz}$.

Two subjects, who already performed the first experiment, were asked to gaze for $60 \mathrm{~s}$ to each of the three patches while the EEG signals were saved on a file and tagged with the corresponding patch identifier.

During another experimental session a different couple of stimulation frequencies were tested as well, corresponding to $12 \mathrm{~Hz}$ and $15 \mathrm{~Hz}$.

\subsection{Results}

The PSD of the recorded files was computed as the Fourier Transform of the $60 \mathrm{~s}$ long signal auto-correlation, in order to highlight the contained frequency components.

As can be appreciated from Fig. 2, the PSD of the signal coming from one of the electrodes (Oz in this case), as expected, reports a clear peak at $15 \mathrm{~Hz}$ and $20 \mathrm{~Hz}$ while the subject was gazing at the left and the right patches respectively.

On the other side, from Fig. 3 it can be appreciated that the SSVEP response elicited by the dichoptic stimulus presented in the center of the screen, contains different peaks in the PSD not present before.

These peaks denote a nonlinear interaction between the SSVEP elicited by the two different flickering frequencies, occurring only when both the frequencies are spatially superimposed. Interestingly, from Fig. 3, it can be noticed that some peaks, as the $20 \mathrm{~Hz}$ one, seems to have been suppressed, although its $10 \mathrm{~Hz}$ subharmonic and its $40 \mathrm{~Hz}$ harmonic are clearly present.

From the reported graphs, the PSD of the SSVEP responses elicited by the three different stimuli showed to the subject are clearly different and thus a classification seems to be feasible. 

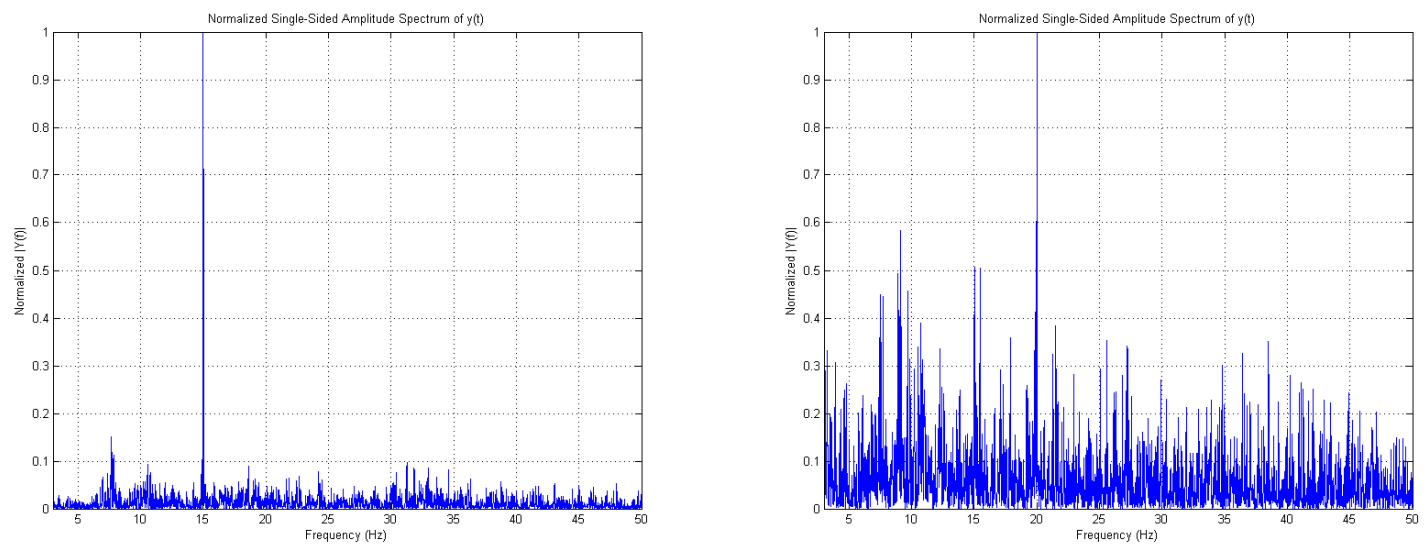

Figure 2. The normalized PSD of the EEG signal acquired by the Oz electrode while the subject was attending a flickering white patch. The observed patch was the left one (flickering at $15 \mathrm{~Hz}$ ), on the left of the image, while it was the right one (flickering at $20 \mathrm{~Hz}$ ), on the right of the image. The apparent different intensity of the background EEG is due to the normalization, since for this subject/electrode/stimulus combination the $20 \mathrm{~Hz}$ peak is weaker than the $15 \mathrm{~Hz}$ one.

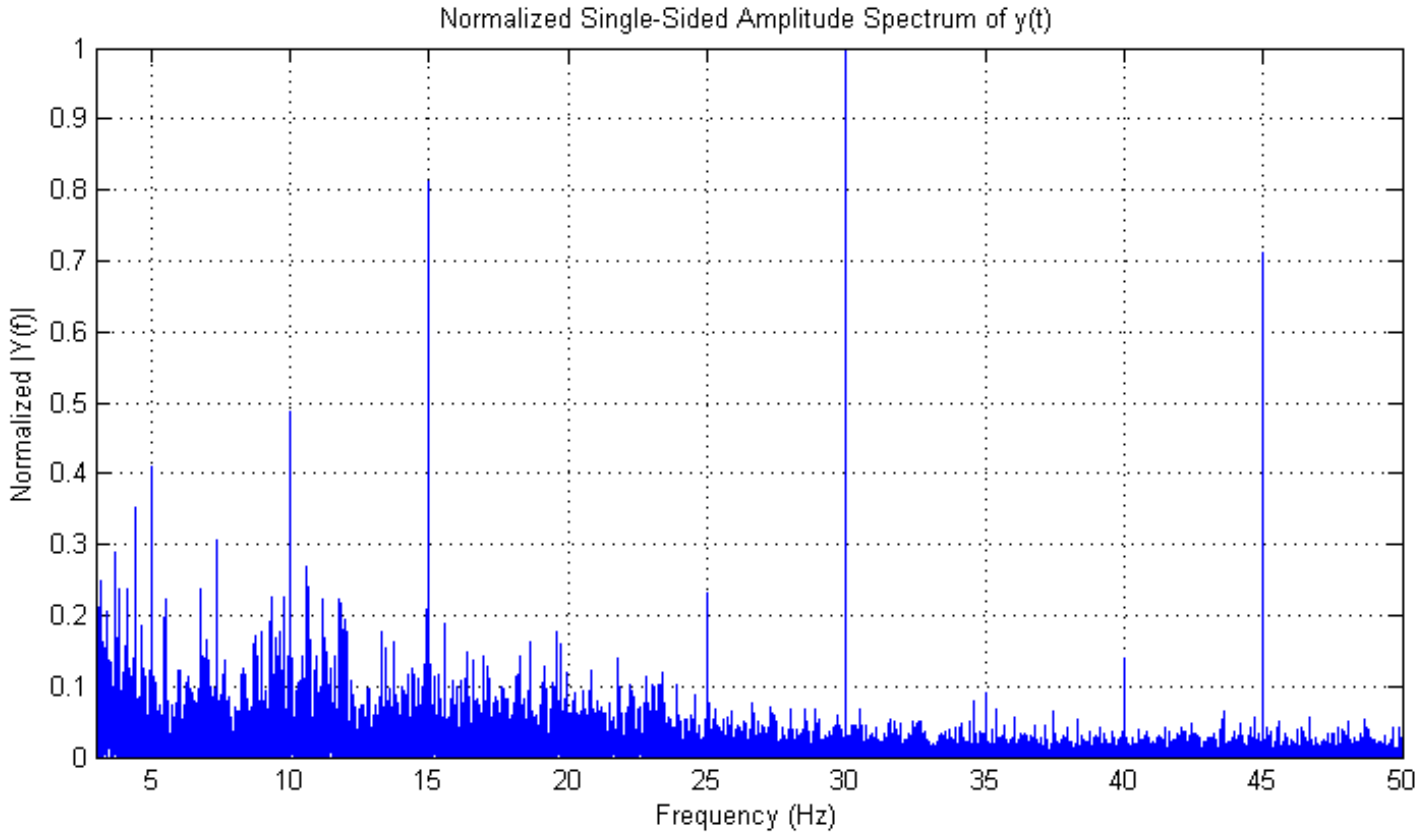

Figure 3. The normalized PSD of the EEG signal acquired by the Oz electrode while the subject was attending a dichoptic flickering white patch with stereoscopic disparity set to zero. The image presented to the left eye was flickering at $15 \mathrm{~Hz}$ while the one presented to the right eye was flickering at $20 \mathrm{~Hz}$.

Moreover, also using different stimulation frequencies, similar observations hold, as shown in Fig. 4, where is reported the PSD of the EEG signal containing a SSVEP responses elicited by a dichoptic flickering stimulus, where the left and right views where respectively flickering at $12 \mathrm{~Hz}$ and $15 \mathrm{~Hz}$.

Also in this condition new peaks which are not present for regular (non-dichoptic) $12 \mathrm{~Hz}$ and $15 \mathrm{~Hz}$ flickering stimuli are present and is particularly prominent a $18 \mathrm{~Hz}$ peak, second harmonic of another strong $9 \mathrm{~Hz}$ peak.

These new peaks, appearing when attending a dichoptic flickering stimulus, have been found in the PSD of the EEG signal recorded from both of the tested subjects at the same frequencies, although with different relative intensities. 


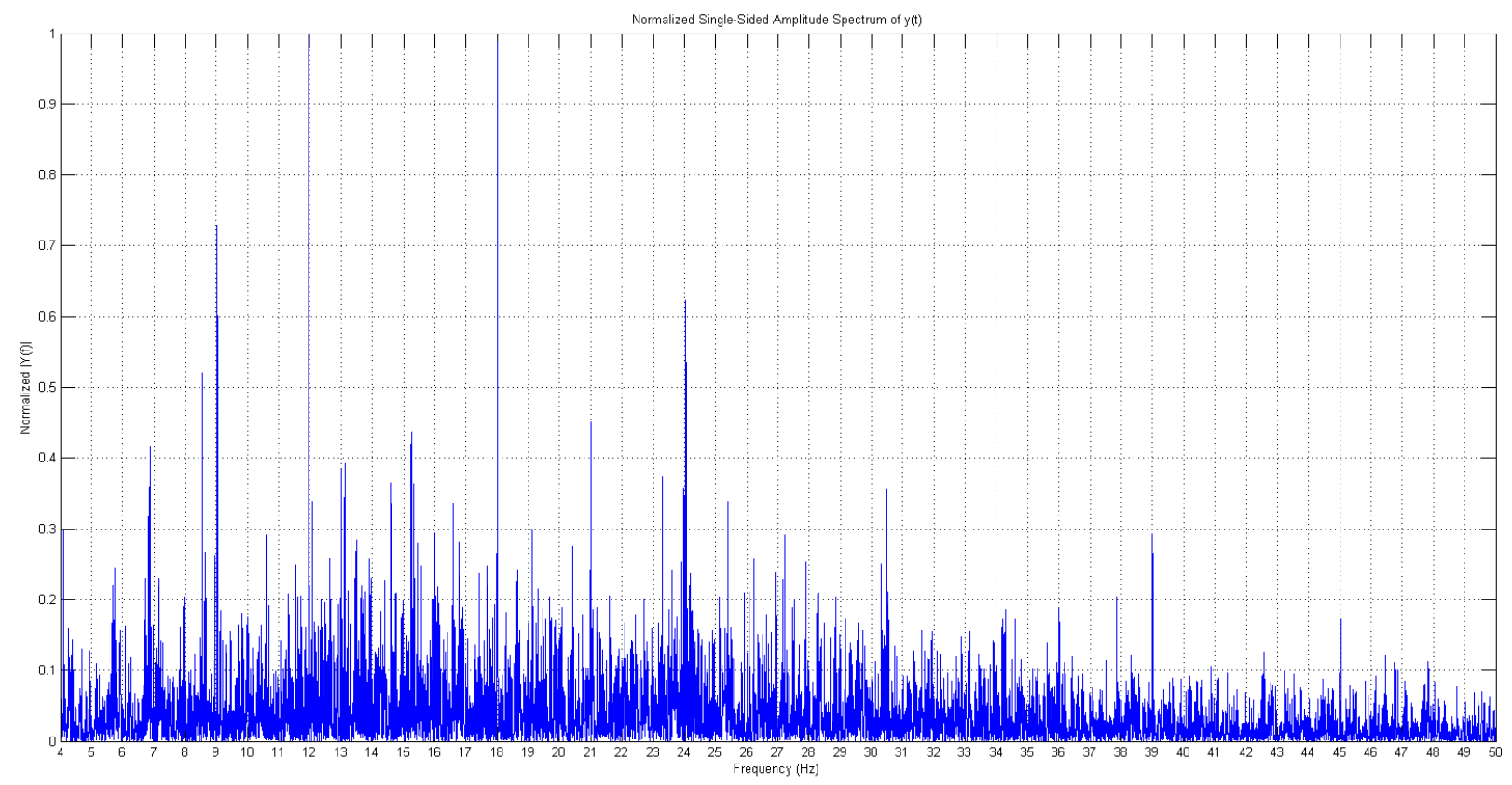

Figure 4. The normalized PSD of the EEG signal acquired by the Oz electrode while the subject was attending a dichoptic flickering white patch with stereoscopic disparity set to zero. The image presented to the left eye was flickering at $12 \mathrm{~Hz}$ while the one presented to the right eye was flickering at $15 \mathrm{~Hz}$.

\section{CONCLUSION AND FUTURE WORKS}

From the first discussed experiment can be inferred that the stereoscopic disparity of a flickering stimulus do not interfere significantly with the elicited SSVEP response, at least for the considered kind of stimulus, which is a commonly used unpatterned white patch. Consequently, prospective SSVEP-based BCI applications in the context of stereoscopic VR environments, could implement integrated flickering stimuli also in objects having a non-zero stereoscopic disparity, without affecting the SSVEP response strength.

Moreover, the used display implements an active sterescopic technology where the user has to wear shuttering glasses, thus the first experiment, as well as the graphs reported in Fig. 2, highlights the fact that the synchronization between the screen refresh and the glasses is precise enough to not introduce unwanted flickers at spurious frequencies nor frequency beatings.

A further interesting experiment would be to compare the performance in terms of the elicited SSVEP response between an active stereoscopic display and a passive one. In fact, although higher frequencies seem not to have been introduced in the PSD of the signals, the shuttering glasses anyway block alternatively the light entering in each eye at a frequency of $60 \mathrm{~Hz}$, thus this may affect the duty cycle of the provided stimulus, with a possible effect on the SSVEP response.

On the other side, the second experiment, in addition to confirming the usability of active stereoscopic displays to provide reliable SSVEP stimuli, demonstrates also how these devices could be used to provide dichoptic SSVEP stimuli. The reported graphs highlight the possibility of implementing three different targets using only two different flickering frequencies, which is an interesting result considering the limited set of properly displayable frequencies on computer monitor devices.

Further experiments will be performed on multiple subjects in order to highlight the inter-subject variability of the nonlinear components elicited by the dichoptic stimulation.

Moreover, on the produced data sets will later be performed an off-line classification in order to determine the shortest signal epoch necessary to obtain an accuracy high enough for SSVEP-based BCI applications. 
Eventually, the dichoptic stimulation used in the second experiment could find applications also in other research fields. In particular, the custom stimuli presentation software ${ }^{16}$, being able to independently control the flickering images displayed in the two eye views and their respective flickering frequencies, thanks to commodity stereoscopic display devices, could find applications in all of the research fields where experiments exploiting the SSVEP-based binocular rivalry paradigm ${ }^{\S}$ are commonly performed ${ }^{5,24}$.

\section{REFERENCES}

1. J. R. Wolpaw, N. Birbaumer, D. J. McFarland, G. Pfurtscheller, T. M. Vaughan, et al., "Brain-computer interfaces for communication and control," Clinical neurophysiology 113(6), pp. 767-791, 2002.

2. F. Lotte, J. Faller, C. Guger, Y. Renard, G. Pfurtscheller, A. Lécuyer, and R. Leeb, "Combining BCI with virtual reality: Towards new applications and improved BCI," in Towards Practical Brain-Computer Interfaces, ch. 10, pp. 197-220, Springer, 2013.

3. Legény Jozef, Abad Raquel Viciana, and Lécuyer Anatole, "Navigating in Virtual Worlds Using a Self-Paced SSVEP-Based Brain-Computer Interface with Integrated Stimulation and Real-Time Feedback," Presence: Teleoperators and Virtual Environments 20(6), pp. 529-544, 2011.

4. D. Regan, "Some early uses of evoked brain responses in investigations of human visual function," Vision research $\mathbf{4 9}(9)$, pp. 882-897, 2009.

5. F.-B. Vialatte, M. Maurice, J. Dauwels, and A. Cichocki, "Steady-state visually evoked potentials: focus on essential paradigms and future perspectives," Progress in neurobiology 90(4), pp. 418-438, 2010.

6. A. Luo and T. J. Sullivan, "A user-friendly SSVEP-based brain-computer interface using a time-domain classifier," Journal of neural engineering 7(2), p. 026010, 2010.

7. C. Guger, B. Z. Allison, B. Grosswindhager, R. Prückl, C. Hintermüller, C. Kapeller, M. Bruckner, G. Krausz, and G. Edlinger, "How many people could use an SSVEP BCI?," Frontiers in Neuroscience 6(169), 2012.

8. A. K. Porbadnigk, S. Scholler, B. Blankertz, A. Ritz, M. Born, R. Scholl, K. Muller, G. Curio, and M. S. Treder, "Revealing the neural response to imperceptible peripheral flicker with machine learning," in Engineering in Medicine and Biology Society, EMBC, 2011 Annual International Conference of the IEEE, pp. 3692-3695, IEEE, 2011.

9. H. Cecotti, I. Volosyak, A. Graser, et al., "Reliable visual stimuli on LCD screens for SSVEP based BCI," in In Proc. of the 18th European Signal Processing Conference (EUSIPCO-2010), 2010.

10. I. P. Howard and B. J. Rogers, Perceiving in Depth, Volume 2: Stereoscopic Vision, no. 29, Oxford University Press, 2012.

11. S. Mun, M.-C. Park, and S. Yano, "Performance Comparison of a SSVEP BCI Task by Individual Stereoscopic 3D Susceptibility," International Journal of Human-Computer Interaction 29(12), pp. 789-797, 2013.

12. M. Cheng, X. Gao, S. Gao, and D. Xu, "Multiple color stimulus induced steady state visual evoked potentials," in Engineering in Medicine and Biology Society, 2001. Proceedings of the 23rd Annual International Conference of the IEEE, 2, pp. 1012-1014, IEEE, 2001.

13. M. Regan and D. Regan, "Objective investigation of visual function using a nondestructive zoom-FFT technique for evoked potential analysis.," The Canadian journal of neurological sciences. Le journal canadien des sciences neurologiques 16(2), p. 168, 1989.

14. V. Zemon, E. Pinkhasov, and J. Gordon, "Electrophysiological tests of neural models: Evidence for nonlinear binocular interactions in humans," Proceedings of the National Academy of Sciences of the United States of America 90(7), pp. 2975-2978, 1993.

15. J. Shils, M. Litt, B. Skolnick, and M. Stecker, "Bispectral analysis of visual interactions in humans," Electroencephalography and Clinical Neurophysiology 98(2), pp. 113-125, 1996.

16. E. Calore, Towards Steady-State Visually Evoked Potentials based Brain-Computer Interfaces for Virtual Reality environments explicit and implicit interaction. PhD thesis, Università degli Studi di Milano, 2014.

\footnotetext{
${ }^{\S}$ Binocular rivalry is a phenomenon of visual perception in which perception alternates between different images presented to each eye. If the two images are flickering at different frequencies, the amplitude of the SSVEP responses at the two frequencies alternates accordingly to the user's perception.
} 
17. Y. Renard, F. Lotte, G. Gibert, M. Congedo, E. Maby, V. Delannoy, O. Bertrand, and A. Lécuyer, "Openvibe: An open-source software platform to design, test, and use brain-computer interfaces in real and virtual environments," Presence: Teleoper. Virtual Environ. 19, pp. 35-53, Feb. 2010.

18. D. Shreiner et al., OpenGL programming guide: the official guide to learning OpenGL, Addison-Wesley Professional, 2009.

19. M. Plong, K. Shen, M. van Vliet, A. Robben, M. Van Hulle, and L. Geurts, "Accurate Visual Stimulus Presentation Software for EEG experiments," in Proceedings of the First Asian Conference on Information Systems, 2012.

20. J. Boyd and Y. Chen, "An open source stimulator for SSVEP-based BCIs," in Proceedings of the 50th Annual Southeast Regional Conference, pp. 124-129, ACM, 2012.

21. D. Zhu, G. G. Molina, V. Mihajlovic, and R. M. Aarts, "Phase synchrony analysis for SSVEP-based BCIs," in Computer Engineering and Technology (ICCET), 2010 2nd International Conference on, 2, pp. V2-329, IEEE, 2010.

22. O. Friman, I. Volosyak, and A. Graser, "Multiple Channel Detection of Steady-State Visual Evoked Potentials for Brain-Computer Interfaces," Biomedical Engineering, IEEE Transactions on 54(4), pp. 742-750, 2007.

23. K. B. Ng, A. P. Bradley, and R. Cunnington, "Stimulus specificity of a steady-state visual-evoked potentialbased brain-computer interface," Journal of Neural Engineering 9(3), 2012.

24. D. Alais and R. Blake, Binocular rivalry, The MIT Press, 2005. 